\title{
Enterovesical Fistula From Meckel Diverticulum
}

\author{
Seung-Rim Han ${ }^{1}$, Hyung-Jin Kim ${ }^{1}$, Ri Na Yoo ${ }^{1}$, Suk Hyun Shin ${ }^{1}$, Gun Kim ${ }^{1}$, Hyeon Min Cho ${ }^{1}$, Seung-Ju Lee ${ }^{2}$, \\ Hyang-Im Lee ${ }^{3}$ \\ ${ }^{1}$ Department of Surgery, St. Vincent's Hospital, College of Medicine, The Catholic University School of Korea, Suwon; ${ }^{2}$ Department of Urology, \\ St. Vincent Hospital, College of Medicine, The Catholic University School of Korea, Suwon; ${ }^{3}$ Department of Pathology, St. Vincent Hospital, \\ College of Medicine, The Catholic University School of Korea, Suwon, Korea
}

Meckel diverticulum is a common congenital malformation of the gastrointestinal tract and can cause complications such as ulceration, hemorrhage, intussusception, and perforation. This report describes a very rare complication of an enterovesical fistula associated with chronic Meckel diverticulum. A 51-year-old male presented with over 10 years of persistent pyuria. Tests were performed to rule out malignancy, including serum prostate-specific antigen level, urine cytology, bacterial culture, cystoscopy, and bladder computed tomography. An enterovesical fistula was identified, and laparoscopic exploration was performed. The findings suggested enterovesical fistula formation caused by chronic inflammation at the tip of a Meckel diverticulum. Segmental resection of the small bowel including the diverticulum and primary repair of the urinary bladder along with partial cystectomy were performed. The postoperative clinical course was uneventful. An enterovesical fistula is a very rare complication resulting from chronic inflammation of a Meckel diverticulum.

Keywords: Enterovesical fistula; Meckel diverticulum; Complication

\section{INTRODUCTION}

An enterovesical fistula (EVF) is an abnormal communication between the intestine and urinary bladder. An EVF can be caused by an inflammatory process, such as Crohn disease or diverticulitis; a malignancy; or surgical trauma. An EVF is a rare complication of Meckel diverticulum, with several reports in the literature [1-7], but only a few cases in Asia [2, 4]. We report this very rare complication of Meckel diverticulum in Korea.

\section{CASE REPORT}

A 51-year-old male was referred to our hospital with a 10-year

Received: Dec 11, 2018 - Revised: Jan 17, 2019 - Accepted: Jan 18, 2019 Correspondence to: Hyung-Jin Kim M.D.

Department of Surgery, St. Vincent's Hospital, College of Medicine, The Catholic University of Korea, 93 Jungbu-daero, Paldal-gu, Suwon 16247, Korea

Tel: +82-31-249-8316, Fax: +82-31-247-5347

E-mail: hj@catholic.ac.kr

ORCID: https://orcid.org/0000-0002-6315-714X

(C) 2021 The Korean Society of Coloproctology

This is an open-access article distributed under the terms of the Creative Commons Attribution NonCommercial License (https://creativecommons.org/licenses/by-nc/4.0) which permits unrestricted noncommercial use, distribution, and reproduction in any medium, provided the original work is properly cited. history of pyuria and increased urinary frequency. Although he had been treated with antibiotics for a chronic urinary tract infection, pyuria persisted without fever or abdominal pain. The patient had hypertension but no significant past surgical history or family history of inflammatory disease.

The prostate was soft and smooth without tenderness. Initial routine blood tests showed no leukocytosis and a normal C-reactive protein level. The urine sediment revealed large numbers of white blood cells and many bacteria. Urine nitrite test was positive, and culture showed growth of Escherichia coli sensitive to a third-generation cephalosporin. Simple abdominal X-ray showed no significant abnormality in the bowel gas pattern or urinary tract. Computed tomography (CT) showed mild, diffuse wall thickening in the urinary bladder with intravesical air density (Fig. 1A). Although adjacent small bowel was observed on the dome of the urinary bladder on coronal CT (Fig. 1B), an EVF could not be identified. Cystoscopy demonstrated a bullous, mass-like lesion at the dome of the urinary bladder, with a hole suggesting a fistula (Fig. 2). Transurethral biopsy was consistent with a vesicointestinal fistula (Fig. 3).

Laparoscopic exploration was performed, and intraoperative findings demonstrated a Meckel diverticulum $(8.5 \times 2.4 \mathrm{~cm})$ attached to the dome of the urinary bladder and omental adhesions. Segmental resection of the small bowel including the 


\section{Coloproctology seung-Rim Han, etal}
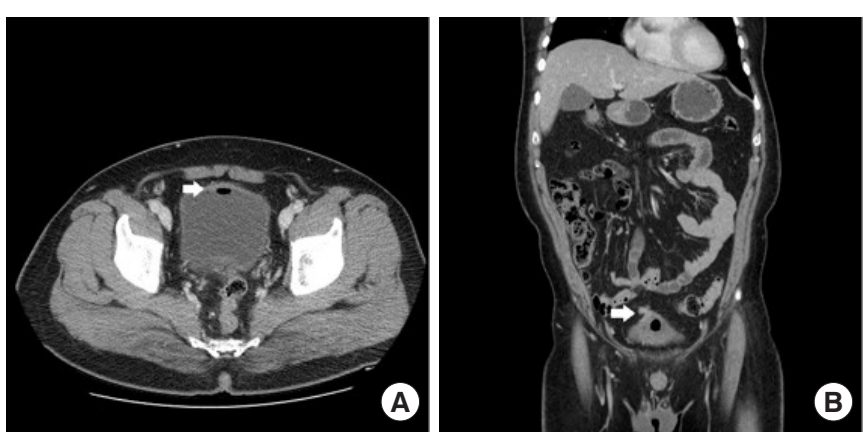

Fig. 1. (A) Mild, diffuse bladder wall thickening with intravesical air density (arrow) in the axial section; (B) adjacent small bowel (arrow) on the dome of the bladder on a coronal section.

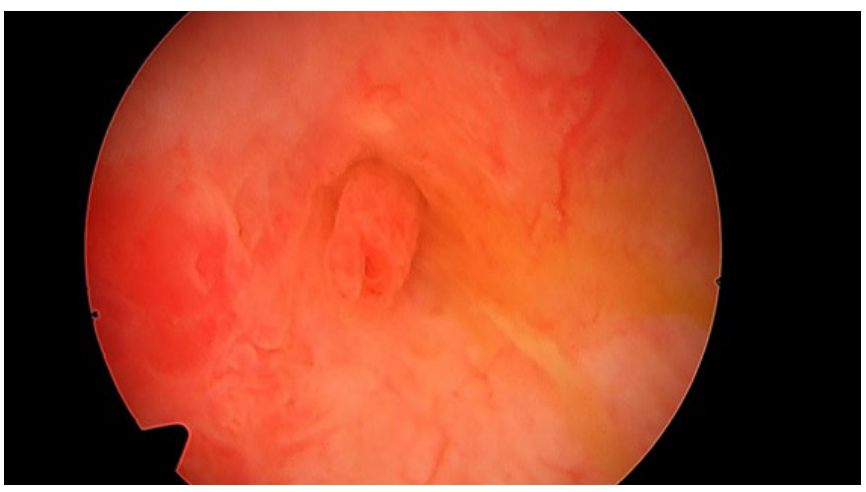

Fig. 2. A fistula opening at the dome of the bladder was detected with cystoscopy.

Meckel diverticulum was performed with partial cystectomy. Macroscopic findings also indicated that the tip of a Meckel diverticulum was connected to the urinary bladder (Fig. 4). The final histologic findings demonstrated a gastric-type Meckel diverticulum with a vesicointestinal fistula.

The postoperative course was uneventful, and the patient was discharged on postoperative day nine without any significant complications. The Institutional Review Board of St. Vincent Hospital approved this case report and waived the informed consent requirement (No. VC18ZOSI-0252).

\section{DISCUSSION}

A Meckel diverticulum, a remnant of the omphalomesenteric duct that connects the midgut of the fetus to the yolk sac, is one of the most common congenital malformations of the gastrointestinal tract. It is reportedly present in $2 \%-4 \%$ of the population [8], with symptoms appearing in only $4 \%-6 \%$ of cases [9]. Gastrointestinal bleeding is the most common presentation of Meckel diverticulum and usually occurs in children, especially those younger than 2 years [8]. In adults, intestinal obstruction due to intussusception or an adhesive band is the most frequent compli-

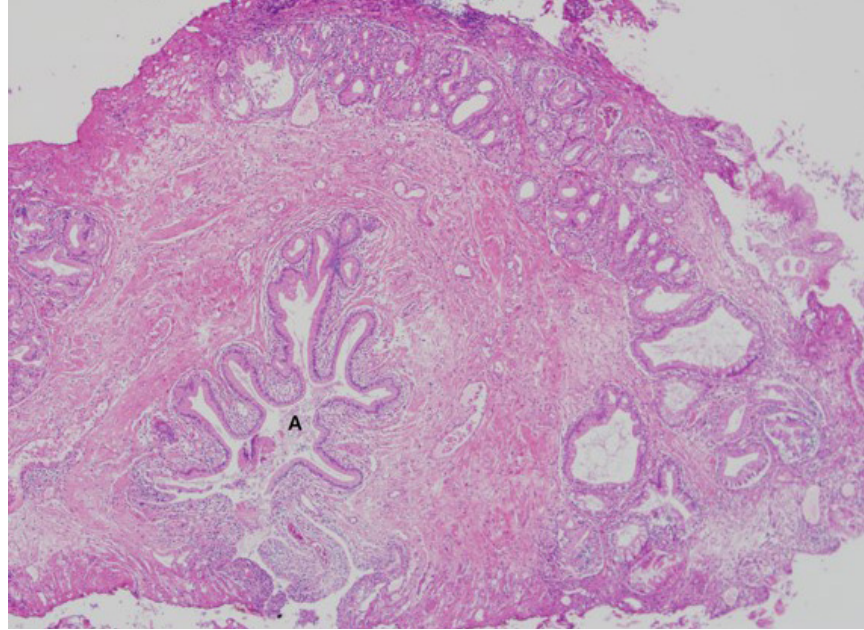

Fig. 3. A specimen from a transurethral bladder resection showing foveolar-type gastric epithelium $(\mathrm{A})$ in the submucosal layer, which is consistent with a vesicointestinal fistula $(\mathrm{H} \& \mathrm{E}, \times 40)$.

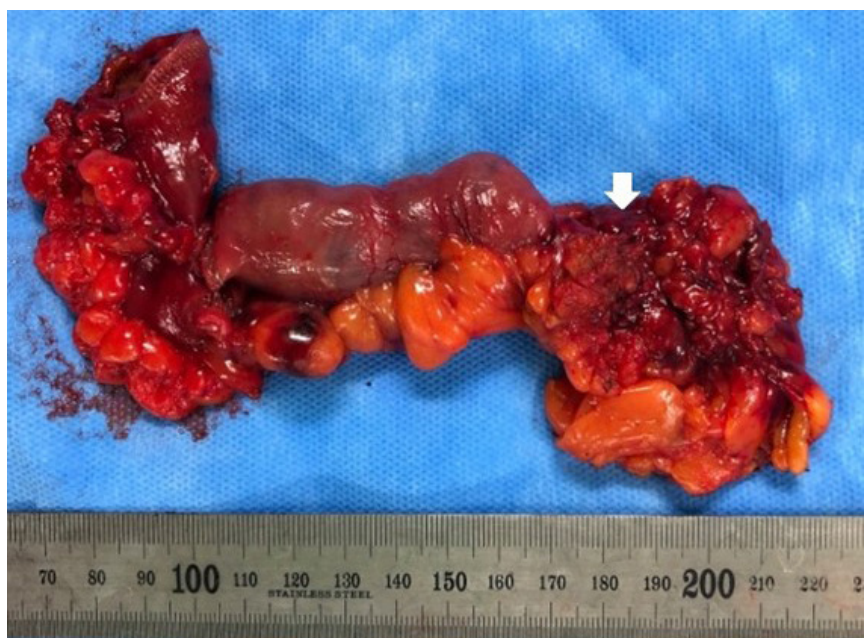

Fig. 4. A specimen from small bowel segmental resection with partial cystectomy: the arrow indicates the location of the enterovesical fistula between the Meckel diverticulum and the urinary bladder.

cation of Meckel diverticulum, accounting for $14 \%-53 \%$ of complicated cases, followed by diverticulitis, enterolith formation, and perforation $[8,10]$. A neoplasm, such as a carcinoid, sarcoma, or adenocarcinoma, accounts for up to $3 \%$ of complicated cases [11].

There are few reports of an EVF associated with Meckel diverticulum. A PubMed and Embase search for articles published between 1990 and 2018 using terms of "enterovesical fistula," "vesicoenteric fistula," and "Meckel's diverticulum" identified seven cases of fistula in the English literature [1-6, 12]. The causes were no coexisting bowel or bladder disease ( 3 cases), adenocarcinoma in ectopic pancreatic tissue (1 case), Crohn disease (1 case), enterolith (1 case), and ingested foreign body in Meckel diverticu- 
Table 1. Published case reports of enterovesical fistula associated with Meckel diverticulum

\begin{tabular}{lllllll}
\hline Study & $\begin{array}{c}\text { Publication year } \\
\text { (country) }\end{array}$ & Sex & Age (yr) & \multicolumn{1}{c}{ Chief complaint } & Type & $\begin{array}{c}\text { Preoperative confirmation } \\
\text { of diagnosis }\end{array}$ \\
\hline Petros et al. [12] & 1990 (United States) & Male & 22 & Fever, dysuria & Gastric & Confirmed with cystography \\
Hudson et al. [5] & 1992 (United States) & Male & 23 & Abdominal pain, diarrhea & No ectopic mucosa & Suspected on CT \\
Graziotti et al. [3] & 2002 (Italy) & Male & 40 & Recurrent dysuria, fever & - & Confirmed with cystoscopic biopsy \\
Bouassida et al. [1] & 2013 (Tunisia) & Female & 66 & Fever, dysuria & No ectopic mucosa & Suspected on cystoscopy \\
Bourguiba et al. [6] & 2017 (Tunisia) & Male & 35 & Fever, abdominal pain with distension & - & No laparotomy \\
Fujita et al. [2] & 2018 (Japan) & Male & 58 & Dysuria, frequency & Pancreatic & Confirmed with cystoscopic biopsy \\
Hakoda et al. [4] & 2018 (Japan) & Male & 46 & Hematuria & No ectopic mucosa & Suspected on cystoscopy, CT, MRI \\
\hline
\end{tabular}

$\mathrm{CT}$, computed tomography; MRI, magnetic resonance imaging.

lum (1 case) (Table 1).

Preoperative diagnosis of Meckel diverticulum is difficult. One study reported that, of 776 patients diagnosed with Meckel diverticulum, only $11 \%$ had symptoms other than bleeding [13]. A preoperative diagnosis of EVF is also challenging. Magnetic resonance imaging and CT have contributed to improved diagnosis of EVF. These modalities are highly effective in determining bladder wall thickness, presence of gas in the bladder, and continuity of a bowel segment adjacent to the bladder. Cystoscopy may also help diagnose EVF through direct visualization of a fistula in $6.7 \%-$ $67 \%$ of cases [7]. This wide variation in detection rate with cystoscopy may be associated with the presence of mucosal edema and hyperplasia, which can cause difficulty in identifying a small fistula orifice [1]. Before visiting our hospital, this patient had undergone cystoscopy at two other hospitals, but no EVF was detected.

Herein, we report the first case of EVF involving a gastric-type Meckel diverticulum in the Asia-Pacific region. Although such a case is very rare and is difficult to diagnose preoperatively, surgeons should be aware of this possibility to avoid a missed or delayed diagnosis.

\section{CONFLICT OF INTEREST}

No potential conflict of interest related to this article was reported.

\section{REFERENCES}

1. Bouassida M, Mighri MM, Trigui K, Chtourou MF, Sassi S, Feidi B, et al. Meckel's diverticulum: an exceptional cause of vesicoenteric fistula: case report and literature review. Pan Afr Med J 2013;15:9.

2. Fujita N, Tambo M, Terado Y, Fujita M, Okegawa T, Nutahara K. Vesicoenteric Fistula arising from an adenocarcinoma of ectopic pancreatic tissue in a Meckel diverticulum. Case Rep Oncol 2018; 11:6-10.

3. Graziotti P, Maffezzini M, Candiano G, Maugeri O. Vesicoenteric fistula created by ingested foreign body in Meckel's diverticulum. J Urol 2002;168:2547.

4. Hakoda H, Mishima H, Habu T, Murai S, Maeno R, Yokomizo Y, et al. Laparoscopic treatment of a vesicointestinal fistula due to a Meckel's diverticulum: a case report and review of the literature. Clin J Gastroenterol 2018;11:476-80.

5. Hudson HM 2nd, Millham FH, Dennis R. Vesico-diverticular fistula: a rare complication of Meckel's diverticulum. Am Surg 1992; 58:784-6.

6. Bourguiba MA, Gharbi M, Ghalleb M, Ben Taher A, Souai F, Bensafta Y, et al. Enterovesical fistula, a rare complication of Meckel's diverticulum: a case report. Int J Surg Case Rep 2017;37: 254-6.

7. Mannion RA, Cottrell TL, Lloyd FA. Vesicoenteric fistula. Am J Surg 1962;103:116-8.

8. Sagar J, Kumar V, Shah DK. Meckel's diverticulum: a systematic review. J R Soc Med 2006;99:501-5.

9. Cikman O, Kiraz HA, Ozkan OF, Adam G, Celik A, Karaayvaz M. An extremely rare complication of Meckel's diverticulum: enterocutaneous fistulization of umbilical hernia. Arq Bras Cir Dig 2015;28:152-3.

10. Srisajjakul S, Prapaisilp P, Bangchokdee S. Many faces of Meckel's diverticulum and its complications. Jpn J Radiol 2016;34:313-20.

11. Konstantakos AK. Meckel's diverticulum-induced ileocolonic intussusception. Am J Surg 2004;187:557-8.

12. Petros JG, Argy O. Enterovesical fistula from Meckel's diverticulum in a patient with Crohn's ileitis. Dig Dis Sci 1990;35:133-6.

13. Higaki S, Saito Y, Akazawa A, Okamoto T, Hirano A, Takeo Y, et al. Bleeding Meckel's diverticulum in an adult. Hepatogastroenterology 2001;48:1628-30. 